\title{
Critical Exponent for Semilinear Wave Equations with Space-Dependent Potential
}

\author{
By \\ Ryo IKeHAtA $^{1}$, Grozdena Todorova ${ }^{2}$ and Borislav Yordanov $^{2}$ \\ $\left({ }^{1}\right.$ Hiroshima University, Japan and ${ }^{2}$ University of Tennessee, USA)
}

\begin{abstract}
We study the balance between the effect of spatial inhomogeneity of the potential in the dissipative term and the focusing nonlinearity. Sharp critical exponent results will be presented in the case of slow decaying potential.

Key Words and Phrases. Damped wave equation, Subcritical potential, Energy decay, $L^{2}$-decay, Small data, Global existence and blow-up, Finite propagation speed.

2000 Mathematics Subject Classification Numbers. Primary 35L70; Secondary 35L05, 35B33, 35B 40 .
\end{abstract}

\section{Introduction}

We consider the following Cauchy problem for the semilinear damped wave equation

$$
\begin{gathered}
u_{t t}(t, x)-\Delta u(t, x)+V(x) u_{t}(t, x)=|u(t, x)|^{p}, \quad(t, x) \in(0, \infty) \times \boldsymbol{R}^{N}, \\
u(0, x)=\varepsilon u_{0}(x), \quad u_{t}(0, x)=\varepsilon u_{1}(x), \quad x \in \boldsymbol{R}^{N},
\end{gathered}
$$

where $\varepsilon>0,\left(u_{0}, u_{1}\right)$ are compactly supported initial data from the energy space

$$
u_{0} \in H^{1}\left(\boldsymbol{R}^{N}\right), \quad u_{1} \in L^{2}\left(\boldsymbol{R}^{N}\right),
$$

$V \in C\left(\boldsymbol{R}^{N}\right)$ is a potential function specified later, and the power $p$ of the nonlinearity satisfies

$$
1<p<\frac{N+2}{N-2} \quad(N \geq 3), \quad 1<p<+\infty \quad(N=1,2) .
$$

Such equations appear in models for travelling waves in a nonhomogeneous gas with damping that changes with the position. $V(x)$ is referred as a friction coefficient or potential (see Ikawa [6]).

Our interst is focused on the so-called critical exponent $p_{c}(N)$, which is a number defined by the following property:

If $p_{c}(N)<p$, all small data solutions of (1.1)-(1.2) are global; while if $1<p \leq p_{c}(N)$ all solutions of (1.1)-(1.2) with data positive on average blow-up in finite time regardless of the smallness of the data. 
Our goal in this paper is to solve the critical exponent problem for the equation (1.1).

It is well known that if the damping is missing, $V(x)=0$, the critical exponent $p_{w}(N)$ for the wave equation $\left(\partial_{t t}-\Delta\right) u=|u|^{p}$ is the positive root of $(N-1) p^{2}-(N+1) p-2=0$, where $N \geq 2$ is the space dimension $\left(p_{w}(1)=\infty\right.$, see Sideris [29], Rammaha [28]). The proof of this fact, famous as Strauss' conjecture [31], took almost 20 years and the effort of many mathematicians, beginning with John [13], Glassey [3], Sideris [30], Strauss [32], Zhou [42], and ending with Lindblad and Sogge [19], Georgiev, Lindblad and Sogge [2] and Tataru [33]. The number $p_{w}(N)$ is refered as Strauss critical exponent. See also the survey papers of Levine [17] and Deng and Levine [16].

In [34] and [35], Todorova and Yordanov solved the critical exponent problem for the wave equation (1.1) when the potential $V(x)$ is a constant. The main result is that the critical exponent $p_{c}(N)$ of the damped wave equation (1.1) with $V(x)=$ const is exactly $1+2 / N$. The number $p_{c}(N)=$ $1+2 / N$ is the famous Fujita's critical exponent for the heat equation $\partial_{t} v-\Delta v=$ $v^{p}$ (see [1]). More precisely, they prove small data global existence for the damped wave equation (1.1) with $V(x)=$ const and exponent $p>1+2 / N$. If $1<p<1+2 / N$, they prove blow up for all solutions of (1.1) with data positive on average. In [35] the authors prove other results which also indicate parabolic asymptotic profile for solutions of equation (1.1) with constant potential $V(x)$ for large exponent $p>p_{c}(N)$. Later on Zhang [41] prove that the critical exponent $1+2 / N$ belongs to the blow up region. IkehataTanizawa [12] consider the global existence part for noncompactly supported data. There are many related results to the so-called diffusion phenomenon, and we quote some of them below: Han-Milani [4], Ikehata [7], IkehataMiyaoka-Nakatake [8], Ikehata-Nishihara [9], Ikehata-Ohta [11], HosonoOgawa [5], Marcati-Nishihara [20], Nishihara [25, 26], Li-Zhou [18], Narazaki [24], Zhang [41], and the references there in.

In this paper we solve the critical exponent problem for the equation (1.1)(1.2) under natural conditions for the potential $V(x)$. For the sake of simplicity the conditions for the potential $V(x)>0$ are the following:

(A) $V(x) \in C\left(R^{N}\right)$ is a radially symmetric function

$$
V(x) \sim V_{0}(1+|x|)^{-\alpha}, \quad|x| \rightarrow \infty,
$$

with $V_{0}>0$ and $\alpha \in[0,1)$.

In the case of slow decaying potential $0 \leq \alpha<1$ in (A) we prove that the critical exponent for the problem (1.1)-(1.2) is

$$
p_{c}(N, \alpha)=1+\frac{2}{N-\alpha} .
$$


The case of fast decaying potential, namely the exponent $\alpha>1$ in (A). In this case there is no decay of the energy of the linear part of equation (1.1). The result of Mochizuki [22] says that the energy of the linear part of equation (1.1) approaches a non-zero constant as $t \rightarrow \infty$ if $V(x)=O\left(|x|^{-1-\delta}\right)$ with $\delta>0$. In this case we expect that equation (1.1) loses its "parabolicity" asymptotic effects and turns back to the regime of pure wave equation. Respectively, we expect that the critical exponent $p_{c}(N, \alpha)$ of the damped wave equation in the case of fast decaying potential $\alpha>1$ jumps to the critical exponent of the wave equation-Strauss' number $p_{w}(N)$. Namely, $p_{c}(N, \alpha)=$ $p_{w}(N)$ for any $\alpha>1$. The proof of both parts - small data global existence and blow-up part is quite involved and will be presented elsewhere.

The case of critically decaying potential $\alpha=1$ in (A) is very delicate. This case is a transition from asymptotic parabolicity to the pure hyperbolic regime. The energy decay of the linear part of equation (1.1) depends in a very interesting way on the coefficient $V_{0}$ of the potential $V(x)$. Correspondingly, the critical exponent is $1+2 /(N-1)$ for large $V_{0} \geq N$, while for $0<V_{0}<N$ the critical exponent $p_{c}\left(N, V_{0}\right)$ increases when $V_{0} \rightarrow 0$. These results will be presented elsewhere.

In this paper we show further interesting phenomena due to the presence of the damping term. We also derive the exact decay rate for the energy and $L^{2}$ and $L^{p+1}$ norms of solutions in the global existence part for exponents of nonlinearity $p>p_{c}(N, \alpha)$.

To get a sharp result as a critical exponent result we need sharp decay estimates for the linear problem

$$
u_{t t}-\Delta u+V(x) u_{t}=0 .
$$

This problem is quite delicate in the case of space dependent potential. Recently Todorova-Yordanov [36, 37] derived almost an optimal decay for solutions of (1.3). The key idea is that we are able to derive asymptotically a very good approximation for the fundamental solution of the equation (1.3). In this paper, for the global existence part namely, the case $p_{c}(N, \alpha)<p$ we use a modification of the approach in [36], [37]. For the blow up the part of (1.1)-(1.2) namely $1<p \leq p_{c}(N, \alpha)$ we apply the method of the test functions developed by Zhang [39, 40, 41].

Now we are ready to state our main results.

Denote by $X_{1}(0, T):=C\left([0, T) ; H^{1}\left(\boldsymbol{R}^{N}\right)\right) \cap C^{1}\left([0, T) ; L^{2}\left(\boldsymbol{R}^{N}\right)\right)$.

Our small data global existence results read as follows.

Theorem 1.1. Let $V(x)$ satisfy condition $(\mathbf{A}) ; \alpha \in[0,1)$ and $p_{c}(N, \alpha)<p<$ $(N+2) /(N-2)$ for $N \geq 3$; and $p_{c}(N, \alpha)<p<\infty$ for $N=1,2$. Then there exists a number $\varepsilon_{0}>0$ such that for any $0<\varepsilon<\varepsilon_{0}$, problem (1.1)-(1.2) has a 
unique solution $u \in X_{1}(0,+\infty)$ satisfying

$$
\begin{gathered}
\int_{\boldsymbol{R}^{N}} e^{(\kappa-\delta)|x|^{2-\alpha} / t} u(t, x)^{2} d x \leq C t^{-m+\alpha /(2-\alpha)}, \\
\int_{\boldsymbol{R}^{N}} e^{\kappa|x|^{2-\alpha} / t}\left(u_{t}(t, x)^{2}+|\nabla u(t, x)|^{2}\right) d x \leq C t^{-m-1}, \\
\int_{\boldsymbol{R}^{N}} e^{\kappa|x|^{2-\alpha} / t}|u(t, x)|^{p+1} d x \leq C t^{-m-1-\gamma},
\end{gathered}
$$

for large $t \gg 1$, where

$$
\begin{gathered}
\kappa=\frac{(m-\delta) V_{0}}{(2-\alpha)(N-\alpha)}, \\
m=\frac{N-\alpha}{2-\alpha}-2 \delta, \\
\gamma=\frac{(p-1)(N-\alpha)-2}{2-\alpha}-(p-1) \delta>0
\end{gathered}
$$

and $\delta>0$ is a small number.

Corollary 1.1. Under the same assumptions as in Theorem 1.1 the following estimates hold

$$
\begin{gathered}
\int_{\boldsymbol{R}^{N}} u(t, x)^{2} d x \leq C t^{-((N-\alpha) /(2-\alpha)-\alpha /(2-\alpha)-2 \delta)}, \\
\int_{\boldsymbol{R}^{N}}\left(u_{t}(t, x)^{2}+|\nabla u(t, x)|^{2}\right) d x \leq C t^{-((N-\alpha) /(2-\alpha)+1-2 \delta)}, \\
\int_{\boldsymbol{R}^{N}}|u(t, x)|^{p+1} d x \leq C t^{-(p(N-\alpha) /(2-\alpha)-\alpha /(2-\alpha)-(p+1) \delta)}
\end{gathered}
$$

for large $t \gg 1$, and small $\delta>0$.

Another important consequence of the main theorem is the following.

Corollary 1.2. Under the same assumptions as in Theorem 1.1, the solution of (1.1)-(1.2) satisfies

$$
\int_{|x|^{2-\alpha} \geq t^{1+\rho}}\left(u_{t}(t, x)^{2}+|\nabla u(t, x)|^{2}\right) d x \leq C_{R} t^{-m} e^{-\kappa t^{\rho}}
$$

for an arbitrarily fixed $\rho>0$. 
Namely, the decay rate of the energy under consideration in the region $|x|^{2-\alpha} \geq t^{1+\rho}(\rho>0)$, is exponential. This shows parabolic asymptotic profile of solutions of the problem (1.1)-(1.2).

The blowup result in the case when $1<p \leq p_{c}(N, \alpha)$ is as follows.

Theorem 1.2. Let the potential $V(x)$ satisfies the condition $(\mathbf{A}) ; \alpha \in[0,1)$ and $1<p \leq p_{c}(N, \alpha)$. If the initial data $\left(u_{0}, u_{1}\right)$ satisfy

$$
\int_{\boldsymbol{R}^{N}}\left(u_{1}(x)+V(x) u_{0}(x)\right) d x>0
$$

then the solution of problem (1.1)-(1.2) does not exist globally for any $\varepsilon>0$.

Let us conclude with an outline of the paper and a remark about the notations. In section 2 we prove Theorem 1.1 by dividing the proof into several lemmas. Section 3 is devoted to the proof of Theorem 1.2.

\section{Global existence of small amplitude solutions}

Throughout this paper, $\|\cdot\|_{q}$ stands for the usual $L^{q}\left(\boldsymbol{R}^{N}\right)$-norm. For simplicity of notation, in particular, we use $\|\cdot\|$ instead of $\|\cdot\|_{2}$.

The following classical local existence result for problem (1.1)-(1.2) is a simple modification of the result in Strauss [32].

Recall that $X_{1}(0, T)=C\left([0, T) ; H^{1}\left(\boldsymbol{R}^{N}\right)\right) \cap C^{1}\left([0, T) ; L^{2}\left(\boldsymbol{R}^{N}\right)\right) . \quad$ Then, under assumption $(\mathbf{A})$ for the potential $V(x)$, for any compactly supported data $\left(u_{0}, u_{1}\right)$ from the energy space

$$
u_{0} \in H^{1}\left(\boldsymbol{R}^{N}\right), \quad u_{1} \in L^{2}\left(\boldsymbol{R}^{N}\right), \quad \operatorname{supp} u_{i} \subset B(R):=\left\{x \in \boldsymbol{R}^{N}:|x|<R\right\}, i=0,1,
$$

problem (1.1)-(1.2) admits a unique solution $u \in X_{1}\left(0, T_{m}\right)$ for some $T_{m}=$ $T_{m}(\varepsilon) \in(0,+\infty]$; if $T_{m}<+\infty$, then

$$
\underset{t \uparrow T_{m}}{\limsup }\left(\left\|u_{t}(t, \cdot)\right\|+\|\nabla u(t, \cdot)\|\right)=+\infty .
$$

Moreover, the finite propagation speed property holds:

$$
\operatorname{supp} u(t, \cdot) \subset B(R+t), \quad t \in\left[0, T_{m}\right) .
$$

It follows from the above local existence that $T_{m}=+\infty$ if

$$
E_{u}(t)=\frac{1}{2}\left(\left\|u_{t}(t, \cdot)\right\|^{2}+\|\nabla u(t, \cdot)\|^{2}\right)
$$

is bounded on every compact subset of $\left[0, T_{m}\right)$. Hence our goal is to show that $E_{u}(t)$, or another equivalent norm, does not blow up in finite time. The proof is split into two parts. 
We begin with the observation that $T_{m} \rightarrow \infty$ as $\varepsilon \rightarrow 0^{+}$. This result is derived from the standard energy inequality, associated with problem (1.1)(1.2):

$$
E_{u}(t) \leq E_{u}(0)+\frac{1}{p+1}\|u(t, \cdot)\|_{p+1}^{p+1}
$$

For completeness we give a lower bound for $T_{m}$ and provide a short proof.

Lemma 2.1. Under the assumptions of Theorem 1.1, the lifespan $T_{m}$ and energy $E_{u}(t)$ satisfy

$$
\begin{gathered}
T_{m}>C_{2} \varepsilon^{-(p-1) /(1-\theta)(p+1)}-R, \\
E_{u}(t) \leq 2 C_{1} \varepsilon^{2}, \quad t \in\left[0, C_{2} \varepsilon^{-(p-1) /(1-\theta)(p+1)}-R\right],
\end{gathered}
$$

where $\theta=N(p-1) / 2(p+1)$ and $C_{1}, C_{2}>0$ depend on $N$, $p$, and $\left(u_{0}, u_{1}\right)$. Thus, $T_{m} \rightarrow \infty$ as $\varepsilon \rightarrow 0^{+}$.

Proof. By using the Gagliardo-Nirenberg and Poincaré inequalities, finite propagation speed and (2.2) we get

$$
\begin{aligned}
E_{u}(t) & \leq E_{u}(0)+C\|\nabla u(t, \cdot)\|^{\theta(p+1)}\|u(t, \cdot)\|^{(1-\theta)(p+1)} \\
& \leq E_{u}(0)+C(1+t+R)^{(1-\theta)(p+1)}\|\nabla u(t, \cdot)\|^{p+1} .
\end{aligned}
$$

Thus we have

$$
E_{u}(t) \leq E_{u}(0)+C(t+R)^{(1-\theta)(p+1)} E_{u}(t)^{(p+1) / 2},
$$

where $C>0$ depends on $N$ and $p$. Expressing $E_{u}(0)$ in terms of the initial data,

$$
E_{u}(t) \leq C_{1} \varepsilon^{2}+C(t+R)^{(1-\theta)(p+1)} E_{u}(t)^{(p+1) / 2}
$$

with $C_{1}=\left(\left\|u_{1}\right\|^{2}+\left\|\nabla u_{0}\right\|^{2}\right) / 2$. Denote by $T$ the first time, such that $E_{u}(T)=$ $2 C_{1} \varepsilon^{2}$. Since $E_{u}(t)$ is continuous and $E_{u}(0)<2 C_{1} \varepsilon^{2}$, we conclude that $E_{u}(t)<$ $2 C_{1} \varepsilon^{2}$ for all $t \in[0, T)$. From estimate (2.3) with $t=T$ we have

$$
2 C_{1} \varepsilon^{2} \leq C_{1} \varepsilon^{2}+C(T+R)^{(1-\theta)(p+1)}\left(2 C_{1} \varepsilon^{2}\right)^{(p+1) / 2} .
$$

By solving this inequality with respect to $T$, we find the following lower bound:

$$
T \geq C_{2} \varepsilon^{-(p-1) /(1-\theta)(p+1)}-R,
$$

where $C_{2}>0$ depends on $N, p$, and $\left(u_{0}, u_{1}\right)$. Since $E_{u}(t) \leq 2 C_{1} \varepsilon^{2}$ for $t \in[0, T]$, the lifespan of $u$ satisfies $T_{m}>T$. It is also clear that $T \rightarrow \infty$ as $\varepsilon \rightarrow 0^{+}$. 
We can now assume that $C_{2} \varepsilon^{-(p-1) /(1-\theta)(p+1)}-R<t<T_{m}$, so we deal with arbitrarily large $t$ by choosing sufficiently small $\varepsilon>0$. Here the standard energy $E_{u}(t)$ is not very convenient for precise estimates of $u$; we use a weighted energy instead. A starting point of the proof of global existence of small data solutions fully depends on the previous work due to [36]. Indeed, for solution $u(t, x)$ on $\left[0, T_{m}\right)$ of the problem $(1.1)-(1.2)$ we set $v=u w^{-1}$, where $w$ is an approximate solution of the linear part of $(1.1)-(1.2)$ and can be defined by

$$
w(t, x):=t^{-m} e^{-m_{1} \phi(x) / t} .
$$

The parameters $m, m_{1}$ are determined below. Here $\phi \in C^{2}\left(\boldsymbol{R}^{N}\right)$ is a positive solution for the Poisson equation:

$$
\Delta \phi(x)=V(x), \quad x \in \boldsymbol{R}^{N},
$$

satisfying

$$
\begin{gathered}
\phi_{0}(1+|x|)^{2-\alpha} \leq \phi(x) \leq \phi_{1}(1+|x|)^{2-\alpha}, \\
m(V):=\liminf _{|x| \rightarrow+\infty} \frac{V(x) \phi(x)}{|\nabla \phi(x)|^{2}}>0
\end{gathered}
$$

where $\phi_{i}>0(i=0,1)$ are constants depending on $V_{0}, N$ and $\alpha$. Solutions $\phi(x)$ with the above properties exist in many cases, including the radial potential $V(x)$ which satisfies the condition $(\mathbf{A})$. In the later case $m(V)$ can be calculated explicitly as follows

$$
m(V)=\frac{N-\alpha}{2-\alpha}
$$

(see [37, Proposition 1.1, Example 1.2]). In this case

$$
m:=m(V)-2 \delta ; \quad m_{1}:=m(V)-\delta,
$$

where $\delta>0$ is a small number.

We also set

$$
P(t, x):=\frac{3}{4}\left(\frac{6}{t}+\frac{S(x)}{t^{2}}\right)^{-1} w(t, x)
$$

where

$$
S(x):=(m(V)-\delta) \phi(x) .
$$

Note that such $P$ and $w$ are defined independently of the solution itself, and satisfy the following properties.

Lemma 2.2. Let $\alpha \in[0,1)$ as in condition (A). There exists a large number $t_{0}>0$ such that for $t \geq t_{0}$ the following inequalities hold 
(i) $C_{1} P(t, x) \geq t^{\alpha} w(t, x)$,

(ii) $\left|w_{t}(t, x)\right| \leq C_{1} t^{-\alpha} w(t, x)$,

with some constant $C_{1}>0$.

Proof. We first obtain

$$
\begin{aligned}
P t^{-\alpha} w^{-1} & =\frac{3}{4} \frac{1}{6 t+(m(V)-\delta) \phi(x)} t^{2-\alpha} \\
& \geq \frac{3}{4} t^{2-\alpha} \frac{1}{6 t+C_{0}(1+|x|)^{2-\alpha}} \geq \frac{3}{4} \frac{t^{2-\alpha}}{6 t+C_{0}(1+t+R)^{2-\alpha}}
\end{aligned}
$$

with some constant $C_{0}>0$. Since

$$
\lim _{t \rightarrow+\infty} \frac{t^{2-\alpha}}{6 t+C_{0}(1+t+R)^{2-\alpha}}=\frac{1}{C_{1}}>0
$$

one has the property (i). (ii) is also similar.

From the condition $(\mathbf{A})$ on the potential $V(x)$ it elementarily follows that there exist constants $V_{1}, V_{2}>0$ such that the following pointwise estimate holds:

$$
V_{1}(1+|x|)^{-\alpha} \leq V(x) \leq V_{2}(1+|x|)^{-\alpha} .
$$

It is easy to check that $v=u w^{-1}$ satisfies the transformed equation on $\left[0, T_{m}(\varepsilon)\right)$ :

$$
v_{t t}-\Delta v+\left(V+2 w^{-1} w_{t}\right) v_{t}+\left(-2 w^{-1} \nabla w\right) \cdot \nabla v+Q(t, x) v=w^{p-1}|v|^{p}
$$

where

$$
Q(t, x)=w^{-1}\left(w_{t t}-\Delta w+V w_{t}\right) .
$$

Multiplying both sides of $(2.6)$ by $\left(P v_{t}+w v\right)$, and integrating over $\boldsymbol{R}^{N}$ we have

$$
\begin{aligned}
& \frac{d}{d t} E\left(v_{t}, \nabla v\right)(t)+F(t)+G(t) \\
& \quad=H(t)+\frac{d}{d t}\left\{\frac{1}{p+1} \int_{\boldsymbol{R}^{N}} P w^{p-1}|v|^{p+1} d x\right\}, \quad t \in\left[0, T_{m}(\varepsilon)\right),
\end{aligned}
$$

where the weighted energy $E\left(v_{t}, \nabla v\right)(t)$ is

$$
E\left(v_{t}, \nabla v\right)(t)=\frac{1}{2} \int_{\boldsymbol{R}^{N}}\left\{P\left(v_{t}^{2}+|\nabla v|^{2}\right)+2 w v_{t} v+\left(Q P+w_{t}+V w\right) v^{2}\right\} d x
$$

and 


$$
\begin{gathered}
F(t)=\frac{1}{2} \int_{\boldsymbol{R}^{N}}\left(-P_{t}+2 V P+4 P(\log w)_{t}-2 w\right) v_{t}^{2} d x \\
+\int_{\boldsymbol{R}^{N}}(\nabla P-2 P \nabla \log w) \cdot v_{t} \nabla v d x+\frac{1}{2} \int_{\boldsymbol{R}^{N}}\left(-P_{t}+2 w\right)|\nabla v|^{2} d x \\
G(t)=\frac{1}{2} \int_{\boldsymbol{R}^{N}}\left[Q w-(Q P)_{t}\right] v^{2} d x \\
H(t)=\int_{\boldsymbol{R}^{N}}\left\{w^{p}-\frac{1}{p+1}\left(P w^{p-1}\right)_{t}\right\}|v|^{p+1} d x .
\end{gathered}
$$

Lemma 2.3. Let $\alpha \in[0,1)$ be the exponent of the potential in condition (A). There exists a large number $t_{0}>0$ such that for $t \geq t_{0}$, and for small $\delta>0$ the following estimates hold

(iii) $-P_{t}+w \geq 0$,

(iv) $Q \geq 0, Q_{t} \leq 0, Q w-(Q P)_{t}=Q\left(w-P_{t}\right)-Q_{t} P \geq 0$,

(v) $\left(-P_{t}+2 w\right)\left(-P_{t}+2 V P+4 P(\log w)_{t}-2 w\right) \geq|\nabla P-2 P \nabla \log w|^{2}$.

Proof. The proof is similar to [36, 37], and so we omit it.

Remark 2.1. Based on the Lemma 2.1 by taking $\varepsilon>0$ sufficiently small we can make the lifespan $T_{m}=T_{m}(\varepsilon)$ of the solution of (1.1)-(1.2) large enough such that $T_{m}>C_{2} \varepsilon^{-(p-1) /(1-\theta)(p+1)}-R>t_{0}$, where $t_{0}>0$ is the time determined in Lemmas 2.2 and 2.3 and $C_{2} \varepsilon^{-(p-1) /(1-\theta)(p+1)}-R$ is the lower bound for the lifespan from Lemma 2.1. This gives us an important bound for $E_{u}\left(t_{0}\right)$ which will be used in the sequel

$$
E_{u}\left(t_{0}\right) \leq 2 C_{1} \varepsilon^{2}
$$

By using Lemma 2.3 we see that $F(t)$ in $(2.0)$ is a positive definite quadratic form, therefore $F(t) \geq 0$. Also $G(t) \geq 0$ because of condition (iv). Therefore, after integrating (2.7) over $\left[t_{0}, t\right]$, where $t_{0}<t<T_{m}$, we have

$$
E\left(v_{t}, \nabla v\right)(t) \leq E\left(v_{t}, \nabla v\right)\left(t_{0}\right)+\frac{1}{p+1} \int_{\boldsymbol{R}^{N}} P w^{p-1}|v|^{p+1} d x+\int_{t_{0}}^{t} H(s) d s .
$$

To estimate the second and third terms on the right hand side, we introduce a new functional:

$$
W(t):=\int_{\boldsymbol{R}^{N}} P(t, x)\left(v_{t}^{2}+|\nabla v|^{2}\right) d x+\int_{\boldsymbol{R}^{N}} V(x) w(t, x) v(t, x)^{2} d x
$$

We postpone the proof that $W(t)$ is equivalent to $E_{u}(t)$ at every fixed $t$. In order to proceed, however, we will show that $W(t)$ is bounded by $E_{u}(t)$. 
Lemma 2.4. For each $t \in\left[0, T_{m}\right)$ it is true that

$$
\begin{gathered}
\int_{\boldsymbol{R}^{N}} w(t, x) v(t, x)^{2} d x \leq C_{R}(t)\|\nabla u(t, \cdot)\|^{2}, \\
E\left(v_{t}, \nabla v\right)(t) \leq C_{R}(t) E_{u}(t),
\end{gathered}
$$

with some t-dependent constant $C_{R}(t)$ satisfying $\lim _{t \rightarrow+\infty} C_{R}(t)=+\infty$.

The proof is omitted since it elementarily follows form the fact that $v=u / w$, $w=t^{-m} e^{-m_{1} \phi(x) / t}$ and the compact support of the data.

Thus, $W(t)$ is well defined and continuous for $t \in\left[t_{0}, T_{m}\right)$. For the second term of the right hand side of (2.11) we have the following crucial estimate.

Lemma 2.5. Let $V(x)$ satisfies the condition (A) and $\alpha \in[0,1)$. If $p_{c}(N, \alpha)<p$, there is a number $\gamma>0$, defined in Theorem 1.1 as a function of $p, N, \alpha$ and $\delta$, such that

$$
\int_{\boldsymbol{R}^{N}}\left(1+\frac{S(x)}{t}\right) P w^{p-1}|v|^{p+1} d x \leq C t^{-\gamma} W(t)^{(p+1) / 2}, \quad t \in\left[t_{0}, T_{m}\right) .
$$

Proof. From definitions of $w(t, x)$ and $P(t, x)$ we have

$$
P w^{p-1}|v|^{p+1}=\frac{3}{4}\left(\frac{6}{t}+\frac{S}{t^{2}}\right)^{-1} w^{p}|v|^{p+1} \leq C t w^{p}|v|^{p+1},
$$

and

$$
\frac{S(x)}{t} P w^{p-1}|v|^{p+1}=\frac{3}{4} t \frac{S(x)}{t}\left(6+\frac{S(x)}{t}\right)^{-1} w^{p}|v|^{p+1} \leq \frac{3}{4} t w^{p}|v|^{p+1} .
$$

Therefore,

$$
\begin{aligned}
\int_{\boldsymbol{R}^{N}}\left(1+\frac{S(x)}{t}\right) P w^{p-1}|v|^{p+1} d x & \leq C t \int_{\boldsymbol{R}^{N}} w^{p}|v|^{p+1} d x \\
& =C t^{-(p m-1)} \int_{\boldsymbol{R}^{N}} e^{-(p S(x)) / t}|v|^{p+1} d x
\end{aligned}
$$

By setting

$$
\psi(t, x)=\frac{1}{2} \frac{S(x)}{t}, \quad \eta=\frac{2 p}{p+1},
$$

we can rewrite (2.12) in the form

$$
\int_{\boldsymbol{R}^{N}}\left(1+\frac{S(x)}{t}\right) P w^{p-1}|v|^{p+1} d x \leq C t^{-(p m-1)}\left\|e^{-\eta \psi(t, \cdot)} v\right\|_{p+1}^{p+1} .
$$


To estimate the weighted norm $\left\|e^{-\eta \psi(t, \cdot)} v\right\|_{p+1}$ we use Gagliardo-Nirenberg inequality and get

$$
\left\|e^{-\eta \psi(t, \cdot)} v\right\|_{p+1} \leq\left\|e^{-\eta \psi(t, \cdot)} v\right\|^{\theta}\left\|\nabla\left(e^{-\eta \psi(t, \cdot)} v\right)\right\|^{1-\theta},
$$

where

$$
\theta=1-N\left(\frac{1}{2}-\frac{1}{p+1}\right)
$$

Since

$$
\left|\nabla\left(e^{-\eta \psi} v\right)\right|^{2}=\eta^{2} e^{-2 \eta \psi}|\nabla \psi|^{2} v^{2}-2 \eta e^{-2 \eta \psi} v \nabla \psi \cdot \nabla v+e^{-2 \eta \psi}|\nabla v|^{2},
$$

integrating by parts we obtain

$$
\begin{aligned}
2 \eta \int_{\boldsymbol{R}^{N}} e^{-2 \eta \psi} v \nabla v \cdot \nabla \psi d x & =\eta \int_{\boldsymbol{R}^{N}} e^{-2 \eta \psi} \nabla v^{2} \cdot \nabla \psi d x=\eta \int_{\boldsymbol{R}^{N}}\left(e^{-2 \eta \psi} \nabla \psi\right) \cdot \nabla v^{2} d x \\
& =-\eta \int_{\boldsymbol{R}^{N}} v^{2}\left(e^{-2 \eta \psi} \Delta \psi-2 \eta e^{-2 \eta \psi}|\nabla \psi|^{2}\right) d x \\
& =2 \eta^{2} \int_{\boldsymbol{R}^{N}} e^{-2 \eta \psi} v^{2}|\nabla \psi|^{2} d x-\eta \int_{\boldsymbol{R}^{N}} v^{2} e^{-2 \eta \psi} \Delta \psi d x
\end{aligned}
$$

Thus we find

$$
\begin{aligned}
\int_{\boldsymbol{R}^{N}}\left|\nabla\left(e^{-\eta \psi} v\right)\right|^{2} d x & =\int_{\boldsymbol{R}^{N}} e^{-2 \eta \psi}|\nabla v|^{2} d x+\int_{\boldsymbol{R}^{N}} e^{-2 \eta \psi}\left(\eta \Delta \psi-\eta^{2}|\nabla \psi|^{2}\right) v^{2} d x \\
& \leq \int_{\boldsymbol{R}^{N}} e^{-2 \eta \psi}|\nabla v|^{2} d x+\eta \int_{\boldsymbol{R}^{N}} e^{-2 \eta \psi}(\Delta \psi) v^{2} d x
\end{aligned}
$$

Therefore, we can rewrite (2.14), as follows

$$
\left\|e^{-\eta \psi(t)} v\right\|_{p+1} \leq\left\|e^{-\eta \psi(t)} v\right\|^{\theta}\left(\int_{\boldsymbol{R}^{N}} e^{-2 \eta \psi}|\nabla v|^{2} d x+\eta \int_{\boldsymbol{R}^{N}} e^{-2 \eta \psi}(\Delta \psi) v^{2} d x\right)^{(1-\theta) / 2} .
$$

Now we estimate the right hand side of (2.15). From the definition of $\psi(t, x)$ and $\phi(x)$ we get

$$
\Delta \psi(t, x)=\frac{1}{2 t} \Delta S(x)=\frac{m(V)-\delta}{2 t} \Delta \phi(x)=\frac{m(V)-\delta}{2 t} V(x) .
$$

Thus we have

$$
\int_{\boldsymbol{R}^{N}} e^{-2 \eta \psi}(\Delta \psi) v(t, x)^{2} d x=\frac{m(V)-\delta}{2 t} \int_{\boldsymbol{R}^{N}} V(x) e^{-2 \eta \psi(t, x)} v(t, x)^{2} d x
$$

Since 


$$
e^{-2 \eta \psi(t, x)}=e^{-2 p S(x) /(p+1) t}=e^{-S(x) / t} t^{-m} e^{-(p-1) S(x) /(p+1) t} t^{m} \leq t^{m} w(t, x),
$$

we get

$$
\int_{\boldsymbol{R}^{N}} e^{-2 \eta \psi}(\Delta \psi) v(t, x)^{2} d x \leq C t^{m-1} \int_{\boldsymbol{R}^{N}} V(x) w(t, x) v(t, x)^{2} d x .
$$

To estimate the weighted norm $\left\|e^{-\eta \psi(t)} v\right\|$ in (2.15) we use the following decomposition:

$$
\begin{aligned}
e^{-2 \eta \psi(t, x)} v(t, x)^{2} & =e^{-S(x) / t} t^{-m} t^{m} e^{-(S(x)(p-1)) /((p+1) t)} t^{-\alpha /(2-\alpha)} t^{\alpha /(2-\alpha)} v^{2} \\
& =t^{m} w(t, x) v(t, x)^{2}\left(e^{-(S(x)(p-1)) /((p+1) t)} t^{-\alpha /(2-\alpha)}\right) t^{\alpha /(2-\alpha)} .
\end{aligned}
$$

Furthermore, there exists a constant $C>0$ such that for any $x>0$ it is true that

$$
x^{\alpha /(2-\alpha)} \leq C e^{x(p-1) /(p+1)}, \quad \alpha \in[0,1), \quad \frac{\alpha}{2-\alpha}<1 .
$$

So, with a generous constant $C>0$ we have

$$
\begin{aligned}
C e^{-(p-1) S(x) /(p+1) t} t^{-\alpha /(2-\alpha)} & \leq C t^{-\alpha /(2-\alpha)}\left(\frac{S(x)}{t}\right)^{-\alpha /(2-\alpha)} \\
& =C S(x)^{-\alpha /(2-\alpha)}=C(m(V)-\delta)^{-\alpha /(2-\alpha)} \phi(x)^{-\alpha /(2-\alpha)} \\
& \leq C V(x) .
\end{aligned}
$$

This inequality combined with (2.18) implies

$$
e^{-2 \eta \psi(t, x)} v(t, x)^{2} \leq C t^{\alpha /(2-\alpha)+m} w(t, x) v(t, x)^{2} V(x),
$$

which shows the desired estimate

$$
\left\|e^{-\eta \psi(t)} v\right\|^{2} \leq C t^{m+(\alpha /(2-\alpha))} \int_{\boldsymbol{R}^{N}} V(x) w(t, x) v(t, x)^{2} d x .
$$

On the other hand, in order to estimate the quantity $\left\|e^{-\eta \psi(t)} \nabla v\right\|$ of (2.15) we use (2.16). In fact, from the definition of $P$ and $(2.16)$ we see that

$$
\begin{aligned}
e^{-2 \eta \psi(t, x)} & =t^{m} w(t, x) e^{-(p-1) S(x) /(p+1) t}=t^{m} \frac{4}{3}\left(\frac{6}{t}+\frac{S(x)}{t^{2}}\right) P(t, x) e^{-(p-1) S(x) /(p+1) t} \\
& =\frac{4}{3} t^{m-1} P(t, x)\left\{\left(6+\frac{S(x)}{t}\right) e^{-(p-1) S(x) /(p+1) t}\right\} \leq C t^{m-1} P(t, x),
\end{aligned}
$$

where $C>0$ is a constant determined by the fact that the function $x \mapsto$ $(6+x) e^{-K x}(K>0)$ is bounded above, that is, for all $x \geq 0,(6+x) e^{-K x} \leq C$. This implies 


$$
\int_{\boldsymbol{R}^{N}} e^{-2 \eta \psi}|\nabla v|^{2} d x \leq C t^{m-1} \int_{\boldsymbol{R}^{N}} P(t, x)|\nabla v|^{2} d x .
$$

Therefore, by using (2.15), (2.17), (2.19) and (2.20) we have

$$
\begin{aligned}
\left\|e^{-\eta \psi(t)} v\right\|_{p+1}^{p+1} \leq & C\left(t^{m+(\alpha /(2-\alpha))} \int_{\boldsymbol{R}^{N}} V(x) w(t, x) v(t, x)^{2} d x\right)^{(p+1) \theta / 2} \\
& \times\left(t^{(m-1)} \int_{\boldsymbol{R}^{N}} V(x) w(t, x) v(t, x)^{2} d x\right. \\
& \left.\quad+t^{(m-1)} \int_{\boldsymbol{R}^{N}} P(t, x)|\nabla v(t, x)|^{2} d x\right)^{(p+1)(1-\theta) / 2} \\
\leq & C\left(t^{m+(\alpha /(2-\alpha))} W(t)\right)^{(p+1) \theta / 2}\left(t^{(m-1)} W(t)\right)^{(p+1)(1-\theta) / 2} \\
= & C t^{\{(m+\alpha /(2-\alpha)) \theta / 2+(m-1)(1-\theta) / 2\}(p+1)} W(t)^{(p+1) / 2} \quad\left(t>t_{0}\right) .
\end{aligned}
$$

This inequality together with (2.13) gives

$$
\int_{\boldsymbol{R}^{N}}\left(1+\frac{S(x)}{t}\right) P w^{p-1}|v|^{p+1} d x \leq C t^{-\gamma} W(t)^{(p+1) / 2}, \quad t>t_{0},
$$

where

$$
\begin{aligned}
\gamma & :=(p m-1)-\left\{\left(m+\frac{\alpha}{2-\alpha}\right) \frac{\theta}{2}+(m-1) \frac{1-\theta}{2}\right\}(p+1) \\
& =\frac{(p-1)(N-\alpha)-2}{2-\alpha}-(p-1) \delta .
\end{aligned}
$$

Finally, from the assumption $p_{c}(N, \alpha)<p$ we find $\gamma>0$, which implies the desired estimate.

Now by using the result in Lemma 2.5 we are able to estimate the third term of the right hand side of the weighted energy inequality (2.11).

Lemma 2.6. Under the assumptions in Lemma 2.5, we have

$$
\int_{t_{0}}^{t} H(s) d s \leq C \int_{t_{0}}^{t} s^{-1-\gamma} W(s)^{(p+1) / 2} d s, \quad t \in\left[t_{0}, T_{m}\right),
$$

where $\gamma>0$ is determined in Lemma 2.5, while $C>0$ is some constant.

Proof. It follows from the definitions of $P$ and $w$ that

$$
K(t, x):=w^{p}-\frac{1}{p+1}\left(P w^{p-1}\right)_{t}=\frac{1}{p+1} P w^{p-1}\left\{(p+1) \frac{w}{P}-\frac{P_{t}}{P}-(p-1) \frac{w_{t}}{w}\right\} .
$$

Since 


$$
\begin{gathered}
\frac{P_{t}}{P}=\left(\frac{6}{t}+\frac{S(x)}{t^{2}}\right)^{-1}\left(\frac{6}{t^{2}}+\frac{2 S(x)}{t^{3}}\right)+\frac{w_{t}}{w}, \\
\frac{w_{t}}{w}=-\frac{m}{t}+\frac{S(x)}{t^{2}}, \quad \frac{w}{P}=\frac{4}{3}\left(\frac{6}{t}+\frac{S(x)}{t^{2}}\right),
\end{gathered}
$$

one has

$$
\begin{aligned}
K(t, x)=P w^{p-1}\{ & \frac{4}{3}\left(\frac{6}{t}+\frac{S(x)}{t^{2}}\right)-\frac{p}{p+1}\left(\frac{S(x)}{t^{2}}-\frac{m}{t}\right) \\
& \left.-\frac{1}{p+1}\left(\frac{6}{t}+\frac{S(x)}{t^{2}}\right)^{-1}\left(\frac{6}{t^{2}}+\frac{2 S(x)}{t^{3}}\right)\right\} \\
\leq & P w^{p-1}\left\{\frac{4}{3}\left(\frac{6}{t}+\frac{S(x)}{t^{2}}\right)+\frac{p m}{(p+1) t}\right\}=t^{-1} P w^{p-1}\left\{\left(8+\frac{p m}{p+1}\right)+\frac{4}{3} \frac{S(x)}{t}\right\} \\
\leq & C t^{-1} P w^{p-1}\left(1+\frac{S(x)}{t}\right),
\end{aligned}
$$

which implies

$$
w(t, x)^{p}-\frac{1}{p+1}\left(P(t, x) w(t, x)^{p-1}\right)_{t} \leq C t^{-1}\left(1+\frac{S(x)}{t}\right) P(t, x) w(t, x)^{p-1} .
$$

This shows

$$
\begin{aligned}
\int_{t_{0}}^{t} H(s) d s & \leq \int_{t_{0}}^{t} \int_{\boldsymbol{R}^{N}}\left(w(s, x)^{p}-\frac{1}{p+1}\left(P(s, x) w(s, x)^{p-1}\right)_{t}\right)|v(s, x)|^{p+1} d x d s \\
& \leq C \int_{t_{0}}^{t} s^{-1} \int_{\boldsymbol{R}^{N}}\left(1+\frac{S(x)}{s}\right) P(s, x) w(s, x)^{p-1}|v(s, x)|^{p+1} d x d s .
\end{aligned}
$$

Thus, by using Lemma 2.5 and the estimate (2.21) we derive the estimate in Lemma 2.6.

From Lemmas 2.5, 2.6 and the weighted energy inequality (2.11) we get the following estimate

$$
\begin{aligned}
E\left(v_{t}, \nabla v\right)= & \frac{1}{2} \int_{\boldsymbol{R}^{N}} P(t, x)\left(v_{t}^{2}+|\nabla v|^{2}\right) d x \\
& +\frac{1}{2} \int_{\boldsymbol{R}^{N}}\left[2 w v_{t} v+\left(Q P+w_{t}+V w\right) v^{2}\right] d x \\
\leq & E\left(v_{t}, \nabla v\right)\left(t_{0}\right)+C W(t)^{(p+1) / 2} \\
& +C \int_{t_{0}}^{t} s^{-1-\gamma} W(s)^{(p+1) / 2} d s, \quad t \geq t_{0} .
\end{aligned}
$$


Since $w>0$ satisfies

$$
w_{t t}-\Delta w+V w_{t} \geq 0
$$

(see [37, Proposition 5.3]) and $P>0$, then $Q P \geq 0$. Since $\partial_{t}\left(w v^{2}\right)-w_{t} v^{2}=$ $2 w v v_{t}$, we can rewrite $(2.22)$ as follows

$$
\begin{aligned}
& \frac{1}{2} \int_{\boldsymbol{R}^{N}} P(t, x)\left(v_{t}^{2}+|\nabla v|^{2}\right) d x+\frac{1}{2} \int_{\boldsymbol{R}^{N}} V w v^{2} d x+\frac{1}{2} \frac{d}{d t} \int_{\boldsymbol{R}^{N}}\left(w v^{2}\right) d x \\
& \leq E\left(v_{t}, \nabla v\right)\left(t_{0}\right)+C W(t)^{(p+1) / 2}+C \int_{t_{0}}^{t} s^{-1-\gamma} W(s)^{(p+1) / 2} d s, \quad t \geq t_{0} .
\end{aligned}
$$

We need one more preparation.

Lemma 2.7. Let $\alpha \in[0,1), c_{0}>0, a>0, R>0$ and $E_{0}>0$ be given real numbers, and let $f \in C\left(\left[t_{0}, T_{m}\right)\right)$ be a monotone increasing function. If a function $h \in C^{1}\left(\left[t_{0}, T_{m}\right)\right)$ satisfies the inequality

$$
h^{\prime}(t)+\frac{c_{0}}{(a+t+R)^{\alpha}} h(t) \leq E_{0}+f(t)
$$

then the following estimate holds

$$
h(t) \leq h\left(t_{0}\right)+C\left(E_{0}+f(t)\right)(a+t+R)^{\alpha}, \quad t \geq t_{0},
$$

with some constant $C>0$.

\section{Proof. Since}

$$
\frac{d}{d t}\left\{e^{c_{0}(a+t+R)^{1-\alpha} /(1-\alpha)} h(t)\right\}=e^{c_{0}(a+t+R)^{1-\alpha} /(1-\alpha)}\left(h^{\prime}(t)+\frac{c_{0}}{(a+t+R)^{\alpha}} h(t)\right),
$$

one obtains

$$
\frac{d}{d t}\left\{e^{c_{0}(a+t+R)^{1-\alpha} /(1-\alpha)} h(t)\right\} \leq e^{c_{0}(a+t+R)^{1-\alpha} /(1-\alpha)}\left(E_{0}+f(t)\right) .
$$

By integrating over $\left[t_{0}, t\right]\left(t<T_{m}\right)$ one has

$$
\begin{aligned}
& e^{c_{0}(a+t+R)^{1-\alpha} /(1-\alpha)} h(t) \leq e^{c_{0}\left(a+t_{0}+R\right)^{1-\alpha} /(1-\alpha)} h\left(t_{0}\right) \\
& \quad+E_{0} \int_{t_{0}}^{t} e^{c_{0}(a+s+R)^{1-\alpha} /(1-\alpha)} d s+\int_{t_{0}}^{t} e^{c_{0}(a+s+R)^{1-\alpha} /(1-\alpha)} f(s) d s \\
& \leq e^{c_{0}(a+t+R)^{1-\alpha} /(1-\alpha)} h\left(t_{0}\right)+\left(E_{0}+f(t)\right) \int_{t_{0}}^{t} e^{c_{0}(a+s+R)^{1-\alpha} /(1-\alpha)} d s
\end{aligned}
$$


where one has just used the monotone increasingness of the function $f(t)$. Since

$$
\begin{aligned}
\int_{t_{0}}^{t} e^{c_{0}(a+s+R)^{1-\alpha} /(1-\alpha)} d s & =\int_{a+t_{0}+R}^{a+t+R} e^{C_{1} \tau^{1-\alpha}} d \tau \\
& =\frac{1}{1-\alpha} \int_{\left(a+t_{0}+R\right)^{1-\alpha}}^{(a+t+R)^{1-\alpha}} e^{C_{1} z} z^{\alpha /(1-\alpha)} d z \\
& \leq e^{C_{1}(a+t+R)^{1-\alpha}} \frac{(a+t+R)^{\alpha}}{C_{1}(1-\alpha)}
\end{aligned}
$$

where $C_{1}=c_{0} /(1-\alpha)$, one has the desired estimate.

Let

$$
M(t):=\max _{t_{0} \leq s \leq t} W(s) .
$$

Note that the function $t \mapsto M(t)$ is monotone increasing and continuous in $\left[t_{0}, T_{m}\right)$. To verify the latter property, let $t_{0} \leq t_{1}<t_{2} \leq t_{3}<T_{m}$ and use

$$
\begin{aligned}
0 & \leq M\left(t_{2}\right)-M\left(t_{1}\right) \\
& \leq \sup \left\{\left|W\left(s_{2}\right)-W\left(s_{1}\right)\right|: t_{0} \leq s_{1}<s_{2} \leq t_{3} \text { and } s_{2}-s_{1} \leq t_{2}-t_{1}\right\} .
\end{aligned}
$$

The upper bound goes to 0 as $t_{2}-t_{1} \rightarrow 0$, since $W$ is uniformly continuous on $\left[t_{0}, t_{3}\right]$.

After these results we can prove

Lemma 2.8. Let $\alpha \in[0,1)$. Then the following bound holds

$$
\begin{aligned}
\int_{\boldsymbol{R}^{N}} w v^{2} d x \leq & \left.\int_{\boldsymbol{R}^{N}} w v^{2} d x\right|_{t=t_{0}} \\
& +C\left(E\left(v_{t}, \nabla v\right)\left(t_{0}\right)+M(t)^{(p+1) / 2}\right. \\
& \left.+\int_{t_{0}}^{t} s^{-1-\gamma} W(s)^{(p+1) / 2} d s\right)(1+t+R)^{\alpha}
\end{aligned}
$$

for all $t \in\left[t_{0}, T_{m}\right)$.

Proof. It follows from (2.23) that

$$
\begin{aligned}
& \frac{1}{4} \int_{\boldsymbol{R}^{N}} V(x) w v^{2} d x+\frac{1}{2} \frac{d}{d t} \int_{\boldsymbol{R}^{N}} w v^{2} d x \\
& \leq E\left(v_{t}, \nabla v\right)\left(t_{0}\right)+C W(t)^{(p+1) / 2}+C \int_{t_{0}}^{t} s^{-1-\gamma} W(s)^{(p+1) / 2} d s, \quad t \in\left[t_{0}, T_{m}\right) .
\end{aligned}
$$


Now by using (2.5) and (2.24) we get

$$
\begin{aligned}
& \frac{1}{2} \frac{V_{0}}{(1+t+R)^{\alpha}} \int_{\boldsymbol{R}^{N}} w v^{2} d x+\frac{d}{d t} \int_{\boldsymbol{R}^{N}} w v^{2} d x \\
& \quad \leq 2 E\left(v_{t}, \nabla v\right)\left(t_{0}\right)+C M(t)^{(p+1) / 2}+C \int_{t_{0}}^{t} s^{-1-\gamma} W(s)^{(p+1) / 2} d s, \quad t \in\left[t_{0}, T_{m}\right) .
\end{aligned}
$$

Since the function

$$
t \mapsto C M(t)^{(p+1) / 2}+C \int_{t_{0}}^{t} s^{-1-\gamma} W(s)^{(p+1) / 2} d s
$$

is monotone increasing, we can apply Lemma 2.7 with

$$
\begin{gathered}
h(t)=\int_{\boldsymbol{R}^{N}} w v^{2} d x, \quad E_{0}=2 E\left(v_{t}, \nabla v\right)\left(t_{0}\right), \quad a=1, \quad c_{0}=\frac{V_{0}}{2}, \\
f(t)=C\left(M(t)^{(p+1) / 2}+\int_{t_{0}}^{t} s^{-1-\gamma} W(s)^{(p+1) / 2} d s\right),
\end{gathered}
$$

and obtain the desired estimate.

After these results, we go to the final stage of the proof of Theorem 1.1.

Proof of Theorem 1.1. Let $t_{0}<t<T_{m}$. For any $\sigma>0$, we have

$$
\begin{aligned}
& \left|2 w v_{t} v\right| \leq \sigma t^{\alpha} w v_{t}^{2}+\sigma^{-1} t^{-\alpha} w v^{2} \\
& 2 w v_{t} v \geq-\sigma t^{\alpha} w v_{t}^{2}-\sigma^{-1} t^{-\alpha} w v^{2}
\end{aligned}
$$

Thus from (2.22) we get

$$
\begin{array}{rl}
\int_{\boldsymbol{R}^{N}} & P\left(v_{t}^{2}+|\nabla v|^{2}\right) d x-\int_{\boldsymbol{R}^{N}} \sigma t^{\alpha} w v_{t}^{2} d x \\
& -\sigma^{-1} t^{-\alpha} \int_{\boldsymbol{R}^{N}} w v^{2} d x+\int_{\boldsymbol{R}^{N}}\left(w_{t}+V w\right) v^{2} d x \\
\leq & 2 E\left(v_{t}, \nabla v\right)\left(t_{0}\right)+C W(t)^{(p+1) / 2}+C \int_{t_{0}}^{t} s^{-1-\gamma} W(s)^{(p+1) / 2} d s
\end{array}
$$

This implies

$$
\begin{aligned}
\int_{\boldsymbol{R}^{N}} & \left(P-\sigma t^{\alpha} w\right)\left(v_{t}^{2}+|\nabla v|^{2}\right) d x+\int_{\boldsymbol{R}^{N}}\left(w_{t}+V w-\sigma^{-1} t^{-\alpha} w\right) v^{2} d x \\
& \leq 2 E\left(v_{t}, \nabla v\right)\left(t_{0}\right)+C W(t)^{(p+1) / 2}+C \int_{t_{0}}^{t} s^{-1-\gamma} W(s)^{(p+1) / 2} d s, \quad t \in\left[t_{0}, T_{m}\right) .
\end{aligned}
$$


Because of Lemma 2.2 we have

$$
\begin{aligned}
2 E\left(v_{t}, \nabla v\right)\left(t_{0}\right)+C W(t)^{(p+1) / 2}+C \int_{t_{0}}^{t} s^{-1-\gamma} W(s)^{(p+1) / 2} d s \\
\geq \int_{\boldsymbol{R}^{N}} P\left(1-\sigma C_{1}\right)\left(v_{t}^{2}+|\nabla v|^{2}\right) d x+\int_{\boldsymbol{R}^{N}}\left(V w+\left(w_{t}-\sigma^{-1} t^{-\alpha} w\right)\right) v^{2} d x \\
\geq\left(1-\sigma C_{1}\right) \int_{\boldsymbol{R}^{N}} P\left(v_{t}^{2}+|\nabla v|^{2}\right) d x+\int_{\boldsymbol{R}^{N}} V w v^{2} d x \\
\quad-\left(C_{1}+\sigma^{-1}\right) t^{-\alpha} \int_{\boldsymbol{R}^{N}} w v^{2} d x, \quad t \in\left[t_{0}, T_{m}\right) .
\end{aligned}
$$

This together with Lemma 2.8 yields

$$
\begin{aligned}
& \left(1-\sigma C_{1}\right) \int_{\boldsymbol{R}^{N}} P\left(v_{t}^{2}+|\nabla v|^{2}\right) d x+\int_{\boldsymbol{R}^{N}} V w v^{2} d x \\
& \leq 2 E\left(v_{t}, \nabla v\right)\left(t_{0}\right)+C W(t)^{(p+1) / 2}+C \int_{t_{0}}^{t} s^{-1-\gamma} W(s)^{(p+1) / 2} d s \\
& +C\left(C_{1}+\sigma^{-1}\right) t^{-\alpha}\left\{\left.\int_{\boldsymbol{R}^{N}} w v^{2} d x\right|_{t=t_{0}}+t^{\alpha}\left(E\left(v_{t}, \nabla v\right)\left(t_{0}\right)+M(t)^{(p+1) / 2}\right.\right. \\
& \left.\left.\quad+\int_{t_{0}}^{t} s^{-1-\gamma} W(s)^{(p+1) / 2} d s\right)\right\} \\
& \leq 2 E\left(v_{t}, \nabla v\right)\left(t_{0}\right)+\left.C t^{-\alpha} \int_{\boldsymbol{R}^{N}} w v^{2} d x\right|_{t=t_{0}}+C E\left(v_{t}, \nabla v\right)\left(t_{0}\right) \\
& \quad+C M(t)^{(p+1) / 2}+C \int_{t_{0}}^{t} s^{-1-\gamma} W(s)^{(p+1) / 2} d s, \quad t \in\left[t_{0}, T_{m}\right),
\end{aligned}
$$

where $C=C_{\sigma}>0$ is a constant (for the definition of $M(t)$, see (2.24)). By taking $\sigma>0$ sufficiently small we have

$$
\begin{aligned}
\int_{\boldsymbol{R}^{N}} P\left(v_{t}^{2}+|\nabla v|^{2}\right) d x+\int_{\boldsymbol{R}^{N}} V w v^{2} d x \\
\leq C\left(E\left(v_{t}, \nabla v\right)\left(t_{0}\right)+\left.\int_{\boldsymbol{R}^{N}} w v^{2} d x\right|_{t=t_{0}}\right)+C M(t)^{(p+1) / 2} \\
\quad+C \int_{t_{0}}^{t} s^{-1-\gamma} W(s)^{(p+1) / 2} d s,
\end{aligned}
$$

for all $t \in\left[t_{0}, T_{m}\right)$. This estimate together with Lemma 2.4 gives the following crucial bound: 


$$
W(t) \leq C_{t_{0}} E_{u}\left(t_{0}\right)+C M(t)^{(p+1) / 2}+\frac{t_{0}^{-\gamma}}{\gamma}\left(\max _{t_{0} \leq s \leq t} W(s)\right)^{(p+1) / 2}, \quad t \in\left[t_{0}, T_{m}\right) .
$$

By using the bound (2.10) we know that $E_{u}\left(t_{0}\right) \leq 2 C_{1} \varepsilon^{2}$, so we have the inequality

$$
M(t) \leq C_{t_{0}} \varepsilon^{2}+C M(t)^{(p+1) / 2}, \quad t \in\left[t_{0}, T_{m}\right),
$$

with another constant $C_{t_{0}}$ which also depends on $N, p$ and $\left(u_{0}, u_{1}\right)$. By Lemma 2.4 it is clear that $M\left(t_{0}\right) \leq C^{*} \varepsilon^{2}$, where $C^{*}$ is a constant. By using the above estimate and standard arguments as in [38], upon possible additional decreasing of $\varepsilon>0$, we get

$$
M(t) \leq 2\left(C_{t_{0}}+C^{*}\right) \varepsilon^{2}, \quad t \in\left[t_{0}, T_{m}\right) .
$$

This implies $T_{m}=+\infty$.

Finally, we derive the decay estimates in Theorem 1.1. Indeed, from (2.26) we have

$$
\begin{array}{rl}
\int_{\boldsymbol{R}^{N}} & P(t, x)\left(v_{t}(t, x)^{2}+|\nabla v(t, x)|^{2}\right) d x \\
\quad+\int_{\boldsymbol{R}^{N}} V(x) w(t, x) v(t, x)^{2} d x \leq C \varepsilon^{2}, \quad t \geq t_{0} .
\end{array}
$$

It follows from the definition of $v=u w^{-1}$ that the second term of the left-hand side of (2.27) satisfies the estimate

$$
\int_{\boldsymbol{R}^{N}} V(x) w(t, x)^{-1} u(t, x)^{2} d x \leq t^{m} \int e^{(m-\delta) \phi(x) / t} V(x) u^{2} d x \leq C \varepsilon^{2} .
$$

Further by using the bounds for $\phi(x)$, namely

$$
\phi_{0}(1+|x|)^{2-\alpha} \leq \phi(x) \leq \phi_{1}(1+|x|)^{2-\alpha} \quad \text { for } x \in \boldsymbol{R}^{N}
$$

and (2.5) we get the estimate

$$
\begin{aligned}
V(x) & \geq C(\phi(x))^{-\alpha /(2-\alpha)} \\
& =C t^{-\alpha /(2-\alpha)}\left(\frac{\phi(x)}{t}\right)^{-\alpha /(2-\alpha)} \\
& \geq C t^{-\alpha /(2-\alpha)} e^{-\delta \phi(x) / t},
\end{aligned}
$$

where $C>0$ and $t \geq t_{0}$ is sufficiently large. We complete the decay estimate for $L^{2}$-norm of solution $u$ by substituting this lower bound for $V(x)$ into inequality (2.28). 
Next, we derive the energy decay estimate for the solution $u$ of (1.1)-(1.2).

Notice that

$$
v_{t}^{2}=\left(-w^{-2} w_{t} u+w^{-1} u_{t}\right)^{2} \geq \frac{1}{2} w^{-2} u_{t}^{2}-3 w^{-4} w_{t}^{2} u^{2},
$$

and

$$
|\nabla v|^{2}=\left|-w^{-2} u \nabla w+w^{-1} \nabla u\right|^{2} \geq \frac{1}{2} w^{-2}|\nabla u|^{2}-3 w^{-4}|\nabla w|^{2} u^{2}
$$

Thus we have

$$
\frac{1}{2} P w^{-2}\left(u_{t}^{2}+|\nabla u|^{2}\right) \leq P\left(v_{t}^{2}+|\nabla v|^{2}\right)+3 P w^{-4}\left(w_{t}^{2}+|\nabla w|^{2}\right) u^{2} .
$$

Integrating this inequality over $\boldsymbol{R}^{N}$, and applying (2.27) one obtains

$$
\frac{1}{2} \int_{\boldsymbol{R}^{N}} P w^{-2}\left(u_{t}^{2}+|\nabla u|^{2}\right) d x \leq C \varepsilon^{2}+3 \int_{\boldsymbol{R}^{N}} P w^{-4}\left(w_{t}^{2}+|\nabla w|^{2}\right) u^{2} d x .
$$

It is easy to check that $P$ and $w$ satisfy

$$
P w^{-3}\left(w_{t}^{2}+|\nabla w|^{2}\right) \leq C V(x) .
$$

By using the above inequality and estimates (2.30) and (2.28) we get

$$
\frac{1}{2} \int_{\boldsymbol{R}^{N}} P w^{-2}\left(u_{t}^{2}+|\nabla u|^{2}\right) d x \leq C \varepsilon^{2}+C \int_{\boldsymbol{R}^{N}} w^{-1} V(x) u^{2} d x \leq C \varepsilon^{2} .
$$

This implies the energy decay estimate in Theorem 1.1 .

Finally, from (2.26) and Lemma 2.5 we find that

$$
\int_{\boldsymbol{R}^{N}}\left(\frac{6}{t}+\frac{S(x)}{t^{2}}\right)^{-1} w^{-1}|u|^{p+1} d x \leq C t^{-\gamma}
$$

so that the decay estimate for the $L^{p+1}$ norm as writen in Theorem 1.1 holds.

\section{Blow-up}

In this section we prove the blow-up result of Theorem 1.2. Recall that the power of nonlinearity $|u|^{p}$ is subcritical or critical, i.e. $p \leq p_{c}(N, \alpha)$. We rely on the method of test functions developed by Zhang [39], [40], [41].

Proof of Theorem 1.2. First we find a non-negative $\phi \in C_{0}^{\infty}\left(\boldsymbol{R} \times \boldsymbol{R}^{N}\right)$, such that 


$$
\phi(t, x)= \begin{cases}1, & \text { if }(t, x) \in[-1,1] \times B(R), \\ 0, & \text { if }(t, x) \in\left(\boldsymbol{R} \times \boldsymbol{R}^{N}\right) \backslash([-2,2] \times B(2 R)) .\end{cases}
$$

We can also satisfy the additional condition

$$
\left|D^{2} \phi(t, x)\right|^{4}+|D \phi(t, x)|^{2} \leq C \phi(t, x), \quad(t, x) \in \boldsymbol{R} \times \boldsymbol{R}^{N},
$$

where $D=\left(\partial_{t}, \nabla\right)$ and $C>0$ is some constant; see [41] for the existence of such functions. Then the test function $\phi_{T}$ is defined by

$$
\phi_{T}(t, x)=\phi\left(\frac{t}{T^{2-\alpha}}, \frac{x}{T}\right), \quad(t, x) \in \boldsymbol{R} \times \boldsymbol{R}^{N},
$$

with some large parameter $T$. Let $P_{T}$ be the subset of $\boldsymbol{R} \times \boldsymbol{R}^{N}$ where $\phi_{T}=1$ and

$$
Q_{T}=\left(\operatorname{supp}\left(D^{2} \phi_{T}\right) \cup \operatorname{supp}\left(D \phi_{T}\right)\right) \cap\left([0,+\infty) \times \boldsymbol{R}^{N}\right),
$$

i.e., $Q_{T}$ is the support of derivatives restricted to $t \geq 0$. It is easy to see that

$$
\begin{aligned}
& P_{T} \supset\left\{(t, x): t \leq T^{2-\alpha} \text { and }|x| \leq R T\right\}, \\
& Q_{T} \subset\left\{(t, x): t>T^{2-\alpha} \text { or }|x|>R T\right\} .
\end{aligned}
$$

Assume that a global solution $u$ exists (i.e., $T_{m}=+\infty$ ) when

$$
1<p \leq p_{c}(N, \alpha)=1+2 /(N-\alpha)
$$

and

$$
\int_{\boldsymbol{R}^{N}}\left(V(x) u_{0}(x)+u_{1}(x)\right) d x>0 .
$$

To obtain a contradiction, we multiply the equation (1.1) by $\phi_{T}^{q}$, with $q=$ $2 p /(p-1)$, and integrate by parts over $[0,+\infty) \times \boldsymbol{R}^{N}$ :

$$
\begin{aligned}
\int_{0}^{\infty} \int_{\boldsymbol{R}^{N}} u\left(\partial_{t}^{2} \phi_{T}^{q}-\Delta \phi_{T}^{q}-V \partial_{t} \phi_{T}^{q}\right) d x d t= & \int_{0}^{\infty} \int_{\boldsymbol{R}^{N}}|u|^{p} \phi_{T}^{q} d x d t \\
& +\int_{\boldsymbol{R}^{N}}\left(V u_{0}+u_{1}\right) d x
\end{aligned}
$$

Here we use $\phi_{T}(0, x)=1, \partial_{t} \phi_{T}(0, x)=0$, and the initial conditions on $u$ to evaluate boundary integrals at $t=0$.

Next, we estimate the integral on the left by Hölder's inequality and compare it with the integral on the right side. A straightforward calculation yields 


$$
\begin{aligned}
\left|\partial_{t}^{2} \phi_{T}^{q}-\Delta \phi_{T}^{q}-V \partial_{t} \phi_{T}^{q}\right| \leq & C\left(\phi_{T}^{q-1}\left|D^{2} \phi_{T}\right|+\phi_{T}^{q-2}\left|D \phi_{T}\right|^{2}+V \phi_{T}^{q-1}\left|\partial_{t} \phi_{T}\right|\right) \\
\leq & C\left(T^{2 \alpha-4} \phi_{T}^{q-3 / 4}+T^{-2} \phi_{T}^{q-3 / 4}\right) \\
& +C\left(T^{-2} \phi_{T}^{q-1}+T^{2 \alpha-4} \phi_{T}^{q-1}+T^{\alpha-2} V \phi_{T}^{q-1 / 2}\right)
\end{aligned}
$$

for $(t, x) \in Q_{T}$, while $\partial_{t}^{2} \phi_{T}^{q}-\Delta \phi_{T}^{q}-V \partial_{t} \phi_{T}^{q}$ is identically 0 for $(t, x) \notin Q_{T}$. Since $\alpha \in[0,1)$ and $\phi_{T} \leq C$, the expression is bounded by $C\left(T^{-2} \phi_{T}^{q-1}+T^{\alpha-2} V \phi_{T}^{q-1 / 2}\right)$. Thus, the left side of identity (3.2) satisfies

$$
\begin{aligned}
& \left|\int_{0}^{\infty} \int_{\boldsymbol{R}^{N}} u\left(\partial_{t}^{2} \phi_{T}^{q}-\Delta \phi_{T}^{q}-V \partial_{t} \phi_{T}^{q}\right) d x d t\right| \\
& \quad \leq C \int_{0}^{\infty} \int_{Q_{T}}|u|\left(T^{-2} \phi_{T}^{q-1}+T^{\alpha-2} V \phi_{T}^{q-1 / 2}\right) d x d t \\
& \quad \leq C\left(\int_{0}^{\infty} \int_{Q_{T}}|u|^{p} \phi_{T}^{q} d x d t\right)^{1 / p} I^{(p-1) / p}(T),
\end{aligned}
$$

with

$$
\begin{aligned}
I(T)= & T^{-2 p /(p-1)} \int_{0}^{\infty} \int_{\boldsymbol{R}^{N}} \phi_{T}^{q-p /(p-1)} d x d t \\
& +T^{(\alpha-2) p /(p-1)} \int_{0}^{\infty} \int_{\boldsymbol{R}^{N}} V^{p /(p-1)} \phi_{T}^{q-p /(2(p-1))} d x d t .
\end{aligned}
$$

Notice that in the above identity all exponent of $\phi_{T}$ are positive since $V(x)=$ $V_{0}(1+|x|)^{-\alpha}$ satisfies the condition $(\mathbf{A})$. Hence

$$
\begin{aligned}
I(T) \leq & C T^{-2 p /(p-1)} \int_{0}^{2 T^{2-\alpha}} \int_{|x| \leq 2 R T} d x d t \\
& +C T^{(\alpha-2) p /(p-1)} \int_{0}^{2 T^{2-\alpha}} \int_{|x| \leq 2 R T}(1+|x|)^{-\alpha p /(p-1)} d x d t
\end{aligned}
$$

which readily simplifies to

$$
I(T) \leq C \begin{cases}T^{-2 p /(p-1)+2-\alpha+N}+T^{(\alpha-2) p /(p-1)+2-\alpha}, & \text { if } \alpha p /(p-1)>N \\ T^{-2 p /(p-1)+2-\alpha+N}+T^{(\alpha-2) p /(p-1)+2-\alpha} \log T, & \text { if } \alpha p /(p-1)=N \\ T^{-2 p /(p-1)+2-\alpha+N}+T^{-2 p /(p-1)+2-\alpha+N}, & \text { if } \alpha p /(p-1)<N .\end{cases}
$$

An upper bound is $I(T) \leq C T^{-2 p /(p-1)+2-\alpha+N}$. We can return to (3.3) and write 


$$
\begin{aligned}
& \left|\int_{0}^{\infty} \int_{\boldsymbol{R}^{N}} u\left(\partial_{t}^{2} \phi_{T}^{q}-\Delta \phi_{T}^{q}-V \partial_{t} \phi_{T}^{q}\right) d x d t\right| \\
& \quad \leq C\left(\int_{0}^{\infty} \int_{Q_{T}}|u|^{p} \phi_{T}^{q} d x d t\right)^{1 / p} T^{-2+(2-\alpha+N)(p-1) / p},
\end{aligned}
$$

where $C$ is independent of $T$. Substituting this estimate into (3.2), we have

$$
\begin{aligned}
& \int_{0}^{\infty} \int_{\boldsymbol{R}^{N}}|u|^{p} \phi_{T}^{q} d x d t \\
& \quad \leq C\left(\int_{0}^{\infty} \int_{Q_{T}}|u|^{p} \phi_{T}^{q} d x d t\right)^{1 / p} T^{-2+(2-\alpha+N)(p-1) / p} .
\end{aligned}
$$

Finally, we show that the above inequality can not hold as $T \rightarrow \infty$. If $p \leq p_{c}(N, \alpha)$, then

$$
-2+(2-\alpha+N)(p-1) / p \leq 0
$$

and (3.4) shows that

$$
\left(\int_{0}^{\infty} \int_{P_{T}}|u|^{p} d x d t\right)^{(p-1) / p} \leq C .
$$

Letting $T \rightarrow \infty$ and using (3.1), we conclude that $u \in L^{p}\left([0,+\infty) \times \boldsymbol{R}^{N}\right)$. Hence (3.1) also implies that $\|u\|_{L^{p}\left(Q_{T}\right)} \rightarrow 0$ as $T \rightarrow \infty$. Passing to the limit in (3.4), we obtain $\|u\|_{L^{p}\left([0,+\infty) \times \boldsymbol{R}^{N}\right)} \leq 0$ for any $1<p \leq p_{c}(N, \alpha)$. This is impossible, since $u$ is a non-trivial solution.

Acknowledgement. The work of the first author was supported in part by Grant-in-Aid for Scientific Research (C) 19540184 of JSPS.

\section{References}

[1] Fujita, H., On the blowing up of solutions of the Cauchy problem for $u_{t}=\Delta u+u^{1+\alpha}, \mathrm{J}$. Fac. Sci. Univ. Tokyo Sec. I, 13 (1966), 109-124.

[2] Georgiev, V., Lindblad, H. and Sogge, C., Weighted Strichartz estimates and global existence for semilinear wave equation, Amer. J. Math., 119 (1997), 1291-1319.

[ 3 ] Glassey, R., Existence in the large for $\square u=F(u)$ in two dimensions, Math. Z., 178 (1981), 233-261.

[4] Han, Y. and Milani, A., On the diffusion phenomenon of quasilinear hyperbolic waves, Bull. Sci. Math., 124 (2000), 415-433.

[5] Hosono, T. and Ogawa, T., Large time behavior and $L^{p}-L^{q}$ estimate of solutions of 2dimensional nonlinear damped wave equations, J. Differential Equations, 203 (2004), 82-118.

[6] Ikawa, M., Hyperbolic partial differential equations and wave phenomena, Translations of Math. Monographs, 189, American Math. Soc., Providence, RI, 2000.

[7] Ikehata, R., Diffusion phenomenon for linear dissipative wave equations in an exterior domains, J. Differential Equations, 186 (2002), 633-651. 
[ 8 ] Ikehata, R., Miyaoka, M. and Nakatake, T., Decay estimates of solutions for dissipative wave equations in $\boldsymbol{R}^{N}$ with lower power nonlinearities, J. Math. Soc. Japan, 56 (2004), 365-373.

[9] Ikehata, R. and Nishihara, K., Diffusion phenomenon for second order linear evolution equations, Studia Math., 158 (2003), 153-161.

[10] Ikehata, R., Nishihara, K. and Zhao, H., Global asymptotics of solutions to the Cauchy problem for the damped wave equation with absorption, J. Differential Equations, 226 (2006), 1-29.

[11] Ikehata, R. and Ohta, M., Critical exponents for semilinear dissipative wave equations in $\boldsymbol{R}^{N}$, J. Math. Anal. Appl., 269 (2002), 87-97.

[12] Ikehata, R. and Tanizawa, K., Global existence of solutions for semilinear damped wave equations in $\boldsymbol{R}^{N}$ with noncompactly supported initial data, Nonlinear Anal., 61 (2005), 11891208.

[13] John, F., Blow-up of solutions of nonlinear wave equations in three space dimensions, Manuscripta Math., 28 (1979), 235-268.

[14] Karch, G., Selfsimilar profiles in large time asymptotics of solutions to damped wave equations, Studia Math., 143 (2000), 175-197.

[15] Kawashima, S., Nakao, M. and Ono, K., On the decay property of solutions to the Cauchy problem of the semilinear wave equation with a dissipative term, J. Math. Soc. Japan, 47 (1995), 617-653.

[16] Deng, K. and Levine, H. A., The role of critical exponents in blow-up theorems: the sequel. J. Math. Anal. Appl., 243 (2000), 85-126.

[17] Levine, H. A., The role of critical exponents in blowup theorems. SIAM Rev., 32 (1990), $262-288$.

[18] Li, T. T. and Zhou, Y., Breakdown of solutions to $\square u+u_{t}=u^{1+\alpha}$, Discrete Contin. Dynam. Systems, 1 (1995), 503-520.

[19] Lindblad, H. and Sogge, C., On the small-power semilinear wave equations, Preprint (1995).

[20] Marcati, P. and Nishihara, K., The $L^{p}-L^{q}$ estimates of solutions to one-dimensional damped wave equations and their application to the compressible flow through porous media, $\mathrm{J}$. Differential Equations, 191 (2003), 445-469.

[21] Matsumura, A., On the asymptotic behavior of solutions of semi-linear wave equations, Publ. Res. Inst. Math. Sci., 12 (1976), 169-189.

[22] Mochizuki, K., Scattering theory for wave equations with dissipative terms, Publ. Res. Inst. Math. Sci., 12 (1976/77), 383-390.

[23] Mochizuki, K. and Nakazawa, H., Energy decay and asymptotic behavior of solutions to the wave equations with linear dissipation, Publ. Res. Inst. Math. Sci., 32 (1996), 401-414.

[24] Narazaki, T., $L^{p}-L^{q}$ estimates for damped wave equations and their applications to semilinear problem, J. Math. Soc. Japan, 56 (2004), 585-626.

[25] Nishihara, K., Asymptotic behavior of solutions of quasilinear hyperbolic equations with linear damping, J. Differential Equations, 137 (1997), 384-395.

[26] Nishihara, K., $L^{p}-L^{q}$ estimates to the damped wave equation in 3-dimensional space and their application, Math. Z., 244 (2003), 631-649.

[27] Nishihara, K. and Zhao, H., Decay properties of solutions to the Cauchy problem for the damped wave equation with absorption, J. Math. Anal. Appl., 313 (2006), 598-610.

[28] Rammaha, M., Nonlinear wave equations in high dimensions, Differential equations and applications, 322-326, Ohio University (1988).

[29] Sideris, T., Ph.D. Thesis, Bloomington: Indiana University (1981).

[30] Sideris, T., Nonexistence of global solutions to semilinear wave equations in high dimensions, J. Differential Equations, 52 (1984), 378-406. 
[31] Strauss, W., Nonlinear scattering theory at low energy, J. Funct. Anal., 41 (1981), 110-133.

[32] Strauss, W., Nonlinear wave equations, C.B.M.S. Lecture Notes, 73, Amer. Math. Soc., Providence, RI, 1989.

[33] Tataru, D., Strichartz estimates in the hyperbolic space and global existence for the semilinear wave equation, Preprint (1998).

[34] Todorova, G. and Yordanov, B., Critical exponent for a nonlinear wave equation with damping, C. R. Acad. Sci. Paris Sér. I Math., 330 (2000), 557-562.

[35] Todorova, G. and Yordanov, B., Critical exponent for a nonlinear wave equation with damping, J. Differential Equations, 174 (2001), 464-489.

[36] Todorova, G. and Yordanov, B., Weighted $L^{2}$-estimates for dissipative wave equations with variable coefficients, J. Differential Equations, 246 (2009), 4497-4518.

[37] Todorova, G. and Yordanov, B., Nonlinear dissipative wave equations with potential, AMS Contemp. Math., 426, Amer. Math. Soc., Province, RI, 2007, 317-337.

[38] Weissler, F., Existence and non-existence of global solutions for a semilinear heat equation, Israel J. Math., 38 (1981), 29-40.

[39] Zhang, Qi S., Nonlinear parabolic problems on manifolds, and a nonexistence result for the noncompact Yamabe problem, Electron. Res. Announc. Amer. Math. Soc., 3 (1997), 45-51 (electronic).

[40] Zhang, Qi S., Blow-up results for nonlinear parabolic equations on manifolds, Duke Math. J., 97 (1999), 515-539.

[41] Zhang, Qi S., A blow-up result for a nonlinear wave equation with damping: the critical case, C. R. Acad. Sci. Paris Sér. I Math., 333 (2001), 109-114.

[42] Zhou, Y., Cauchy problem for semilinear wave equations in four space dimensions with small initial data, J. Partial Differential Equations, 8 (1995), 135-144.

\author{
nuna adreso: \\ Ryo Ikehata \\ Department of Mathematics \\ Graduate School of Education \\ Hiroshima University \\ Higashi-Hiroshima 739-8524 \\ Japan \\ E-mail: ikehatar@hiroshima-u.ac.jp \\ Grozdena Todorova \\ Department of Mathematics \\ University of Tennessee \\ Knoxville \\ USA \\ E-mail: todorova@math.utk.edu \\ Borislav Yordanov \\ Department of Mathematics \\ University of Tennessee \\ Knoxville \\ USA \\ E-mail: yordanov@math.utk.edu
}

(Ricevita la 9-an de februaro, 2009) 